QUANTUM PROBABILITY

BANACH CENTER PUBLICATIONS, VOLUME 73

INSTITUTE OF MATHEMATICS

POLISH ACADEMY OF SCIENCES

WARSZAWA 2006

\title{
SOME CLASS OF POLYNOMIAL HYPERGROUPS
}

\author{
WOJCIECH MŁOTKOWSKI \\ Institute of Mathematics, Wrockaw University \\ Pl. Grunwaldzki 2/4, 50-384 Wrockaw, Poland \\ E-mail:mlotkow@math.uni.wroc.pl
}

\begin{abstract}
We provide explicit formulas for linearizing coefficients for some class of orthogonal polynomials.
\end{abstract}

Introduction. Let $\left\{P_{n}\right\}_{n=0}^{\infty}$ be a sequence of monic polynomials, $\operatorname{deg} P_{n}=n$, which are orthogonal with respect to a probability measure $\mu$ on $\mathbf{R}$ with infinite support. Then, under the convention that $P_{-1}=0$, they satisfy the recurrence relation

$$
x P_{n}(x)=P_{n+1}(x)+\beta_{n} P_{n}(x)+\gamma_{n-1} P_{n-1}(x), \quad n \geq 1,
$$

with $P_{0}(x)=1, \gamma_{n}>0$ and $\beta_{m}$ real (see [Ch]). We denote by $\mathcal{L}$ the linear functional on $\mathbf{R}[x]$ given by $\mathcal{L}(P):=\int P(x) d \mu(x)$.

Now we define the linearization coefficients by the relation

$$
P_{n}(x) P_{m}(x)=\sum_{j} c(j, m, n) P_{j}(x) .
$$

We say that $\left\{P_{n}\right\}_{n=0}^{\infty}$ admits nonnegative product linearization if all these coefficients $c(k, m, n)$ are nonnegative. In this case one can define a hypergroup in the following way: Choose $x_{0}$ such that $P_{m}\left(x_{0}\right)>0$ for every $m$ (this holds if and only if $x_{0} \geq \sup (\operatorname{supp} \mu)$ ) and put

$$
\delta_{m} * \delta_{n}:=\sum_{k} \frac{c(k, m, n) P_{k}\left(x_{0}\right)}{P_{m}\left(x_{0}\right) P_{n}\left(x_{0}\right)} \delta_{k} .
$$

Extending this to convex combinations one obtains an associative and commutative operation on the class of probability measures on the set $\{0,1,2, \ldots\}$ (see $[\mathrm{BH}],[\mathrm{Ko}]$ ). Many of the classical orthogonal polynomials do admit nonnegative product linearization (see

2000 Mathematics Subject Classification: 42C05.

Key words and phrases: orthogonal polynomials, linearization coefficients.

Research supported by KBN: 2 P03A 007 23, by RTN: HPRN-CT-2002-00279 and KBNDAAD project $36 / 2003 / 2004$.

The paper is in final form and no version of it will be published elsewhere. 
$[\mathrm{BH}])$. On the other hand, there are some general criteria stated in terms of the coefficients $\beta_{n}, \gamma_{n}$, which imply this property (see [As, Sz1, Sz2, MS]).

The main result. Let us denote $L(k, m, n):=\mathcal{L}\left(P_{k} P_{m} P_{n}\right)$. Then multiplying both sides of $(2)$ by $P_{k}$ and applying $\mathcal{L}$ we get $L(k, m, n)=c(k, m, n) \mathcal{L}\left(P_{k}^{2}\right)$, so from now on we will be studying the numbers $L(k, m, n)$ instead of $c(k, m, n)$. We will be using the following properties:

(3) $L(k, m, k+m)=\gamma_{0} \gamma_{1} \ldots \gamma_{k+m-1}$,

(4) $L\left(k_{1}, k_{2}, k_{3}\right)=L\left(k_{\sigma_{1}}, k_{\sigma_{2}}, k_{\sigma_{3}}\right)$ for every permutation $\sigma$ of the set $\{1,2,3\}$,

$$
L(k, m, n)=0 \quad \text { whenever } n>k+m \text {, }
$$

and

$$
\begin{aligned}
L(k, m, n)= & L(k-1, m, n+1)+\left(\beta_{n}-\beta_{k-1}\right) L(k-1, m, n) \\
& +\gamma_{n-1} L(k-1, m, n-1)-\gamma_{k-2} L(k-2, m, n)
\end{aligned}
$$

for every $k, m, n$.

To prove them we first note that if $\operatorname{deg} P<n$ then $\mathcal{L}\left(P \cdot P_{n}\right)=0$ because $P$ can be expressed as a linear combination of $P_{0}, P_{1}, \ldots, P_{n-1}$. In particular, putting $P=P_{k} P_{m}$ we prove $(5)$.

We use this remark again to note that

$$
\mathcal{L}\left(x P_{k-1} P_{m} P_{k+m}\right)=L(k, m, k+m)
$$

and

$$
\mathcal{L}\left(x P_{k-1} P_{m} P_{k+m}\right)=L(k-1, m+1, k+m),
$$

so we obtain $L(k, m, m+n)=L(k-1, m+1, m+n)$. Hence it is sufficient to prove (3) in the case when $k=0$. Now, by orthogonality we have

$$
\mathcal{L}\left(P_{m} P_{m}\right)=\mathcal{L}\left(x P_{m-1} P_{m}\right)=\gamma_{m-1} \mathcal{L}\left(P_{m-1} P_{m-1}\right),
$$

which completes the proof of (3).

For the last formula one only needs to compare the right hand sides of the equalities:

$$
\begin{aligned}
& \mathcal{L}\left(x P_{k-1} P_{m} P_{n}\right)=L(k, m, n)+\beta_{k-1} L(k-1, m, n)+\gamma_{k-2} L(k-2, m, n), \\
& \mathcal{L}\left(x P_{k-1} P_{m} P_{n}\right)=L(k-1, m, n+1)+\beta_{n} L(k-1, m, n)+\gamma_{n-1} L(k-1, m, n-1) .
\end{aligned}
$$

Note in passing that in view of (6) the coefficients $L(k, m, n)$ can be expressed as a sum of weights of so-called Motzkin paths on the plane connecting points $(0, m)$ and $(k, n)$. Namely, the step from the point $(i-1, j+1),(i-1, j),(i-1, j-1)$ or $(i-2, j)$, respectively, to $(i, j)$ is equal to $1, \beta_{j}-\beta_{i-1}, \gamma_{j-1}$ or $-\gamma_{i-2}$, respectively, and the weight of a path is the product of weights of its steps (see [MS]).

From now on we will assume that

$$
\gamma_{n}=\left\{\begin{array}{ll}
a & \text { if } n=0, \\
b & \text { if } n \text { is odd }, \\
c & \text { if } n>0 \text { is even, }
\end{array} \quad \beta_{n}= \begin{cases}u & \text { if } n=0 \\
v & \text { if } n \text { is odd } \\
w & \text { if } n>0 \text { is even }\end{cases}\right.
$$


This class contains orthogonal polynomials related to some interesting distributions which appear in noncommutative probability (see [BLS]). In the case when $b=c$ and $v=w$ the corresponding measure was thoroughly studied in [SY].

Our aim is to provide explicit formulas for the coefficients $L(k, m, n)$ in this case. Note, that by (4) and (5) we can assume that $k \leq m \leq n \leq k+n$. Set $\Gamma(s):=\prod_{i=0}^{s-1} \gamma_{i}$. If $s>1$ then $\Gamma(s)=\Gamma(s-1) b$ if $s$ is even and $\Gamma(s)=\Gamma(s-1) c$ if $s$ is odd.

Theorem. Assume that $0 \leq k \leq m \leq n \leq k+m$ and put $s:=[(k+m+n) / 2]$. Then $L(k, m, n)$ is equal to:

e1) $\Gamma(s)$ if $n=k+m$,

e2) $\Gamma(s-1)(b+c-a+(w-u)(w-v))$ if $k, m, n$ are even, $s$ is odd,

e3) $\Gamma(s-1)(b+c-a)$ if only one of the numbers $k, m, n$ is even, $s$ is even and $n<k+m$,

e4) $\Gamma(s-1)(2 c-a)$ if $n<k+m$ and either $k, m, n, s$ are all even or only one of the numbers $k, m, n$ is even, $s$ is odd,

o1) $\Gamma(s-1)(a(w-v)+c(v-u))$ if $k, m, n$ are odd, $s$ is even

o2) $\Gamma(s)(v-u)$ if $k, m, n$ are odd, $s$ is odd,

o3) $\Gamma(s)(w-u)$ if only one of the numbers $k, m, n$ is odd.

Note that in cases (e1)-(e4) the sum $k+m+n$ is even, while in cases (o1)-(o3) it is odd.

Proof. We will proceed by induction on $k$. First we examine a few particular cases. For $n=k+m$ the formula is a consequence of (3) so we will assume that $k \geq 1$ and $n<k+m$.

If $k=1 \leq m$ then (6) yields

$$
L(1, m, m)=\left(\beta_{m}-u\right) L(0, m, m)= \begin{cases}\Gamma(m)(v-u) & \text { if } m \text { is odd } \\ \Gamma(m)(w-u) & \text { if } m \text { is even }\end{cases}
$$

which completes the proof for $k=1$.

Now let us consider the special case when $n=k+m-1, k \geq 2$. Then $k+m+n=$ $2(k+m-1)+1$ is odd, $s=k+m-1=n$ and $L(k-1, m, n+1)=L(k-2, m, n)=0$. Moreover, if $m$ is even then $\beta_{n}-\beta_{k-1}=0$ so we get

$$
L(k, m, k+m-1)=\gamma_{k+m-2} \Gamma(k+m-2)(w-u)=\Gamma(k+m-1)(w-u) .
$$

If $m$ is odd, $k$ is even then $n$ is even so

$$
\begin{aligned}
L(k, m, k+m-1) & =(w-v) \Gamma(k+m-1)+b \Gamma(k+m-2)(v-u) \\
& =\Gamma(k+m-1)(w-u),
\end{aligned}
$$

and if $k, m$ are odd then $n$ is odd, hence

$$
\begin{aligned}
L(k, m, k+m-1) & =(v-w) \Gamma(k+m-1)+c \Gamma(k+m-2)(w-u) \\
& =\Gamma(k+m-1)(v-u) .
\end{aligned}
$$

Now let us check the case $k=2$ and $n=m$. If $m$ is even, we have

$$
\begin{aligned}
L(2, m, m) & =L(1, m, m+1)+(w-v) L(1, m, m)+b L(1, m, m-1)-a L(0, m, m) \\
& =\Gamma(m+1)+\Gamma(m)(w-u)(w-v)+\Gamma(m) b-\Gamma(m) a \\
& =\Gamma(m)(c+(w-u)(w-v)+b-a)
\end{aligned}
$$


while if $m$ odd then $\beta_{n}-\beta_{k-1}=v-v=0$ and we get

$$
\begin{aligned}
L(2, m, m) & =L(1, m, m+1)+c L(1, m, m-1)-a \Gamma(0, m, m) \\
& =\Gamma(m+1)+c \Gamma(m)-a \Gamma(m)=\Gamma(m)(b+c-a) .
\end{aligned}
$$

Therefore we have completed the case $k=2$.

From now on we assume that $2<k \leq m<k+m-1$. We will consider four cases.

I. Assume that $k, n$ are even. Then $L(k, m, n)=L(k-1, m, n+1)+(w-v) L(k-1, m, n)+b L(k-1, m, n-1)-c L(k-1, m, n)$.

a) If $m, s$ are even then the sum of the first, second and forth summand is zero and we get

$$
\begin{gathered}
L(k, m, n)=\Gamma(s-1)(b+c-a)+(w-v) \Gamma(s-1)(w-u)+b \Gamma(s-3) b(2 c-a) \\
-c \Gamma(s-2)(b+c-a+(w-u)(w-v))=\Gamma(s-2) b(2 c-a)
\end{gathered}
$$

b) If $m$ is even and $s$ is odd then the first summand cancels with the fourth one (including the special case $n=k+m-2$ ) so $L(k, m, n)=(w-v) \Gamma(s-1)(w-u)+b \Gamma(s-2)(b+c-a)=\Gamma(s-1)(b+c-a+(w-u)(w-v))$.

c) Now assume that $m$ is odd and $s$ is even. Then

$$
\begin{aligned}
& L(k, m, n)=\Gamma(s-1)(a(w-v)+c(v-u))+(w-v) \Gamma(s-1)(b+c-a) \\
& +b \Gamma(s-1)(v-u)-c \Gamma(s-1)(w-u)+\Gamma(s-1) b(w-u)=\Gamma(s)(w-u)
\end{aligned}
$$

d) Finally, assume that $m, s$ are odd. Then

$$
L(k, m, n)=\Gamma(s)(v-u)+(w-v) \Gamma(s-2) b(2 c-a)
$$

$+b \Gamma(s-2)(a(w-v)+c(v-u))-c \Gamma(s-1)(w-u)+\Gamma(s-1) c(w-u)=\Gamma(s)(w-u)$.

II. If $k$ is even and $n$ is odd then $\beta_{n}-\beta_{k-1}=v-v=0, \gamma_{n-1}=\gamma_{k-2}=c$ and $L(k-1, m, n-1)=L(k-2, m, n)$ (unless $n=k+m-2)$, which leads

$$
L(k, m, n)=L(k-1, m, n+1) .
$$

In the case $n=m+k-2$ we get

$$
\begin{aligned}
L(k, m, n+k-2) & =\Gamma(k+m-1)+c \Gamma(k+m-4) b(2 c-a)-c \Gamma(k+m-2) \\
& =\Gamma(k+m-2)(b+c-a) .
\end{aligned}
$$

III. Similarly, if $k$ is odd and $n$ is even then $\beta_{n}-\beta_{k-1}=w-w=0, \gamma_{n-1}=\gamma_{k-2}=b$ and $L(k-1, m, n-1)=L(k-2, m, n)$ (again, unless $n=k+m-2)$, which, similarly as before, leads

$$
L(k, m, n)=L(k-1, m, n+1),
$$

and for $n=k+m-2$ we get

$$
\begin{aligned}
L(k, m, k+m-2) & =\Gamma(k+m-1)+b \Gamma(k+m-3)(b+c-a)-b \Gamma(k+m-2) \\
& =\Gamma(k+m-2)(2 c-a) .
\end{aligned}
$$

IV. Finally, assume that $k, n$ are odd. Then $L(k, m, n)=L(k-1, m, n+1)+(v-w) L(k-1, m, n)+c L(k-1, m, n-1)-b L(k-2, m, n)$. 
a) If $m, s$ are even then, similarly as in the case (Ib), the first summand cancels with the fourth one:

$L(k, m, n)=(v-w) \Gamma(s-1)(w-u)+c \Gamma(s-2)(b+c-a+(w-u)(w-v))=\Gamma(s-1)(b+c-a)$.

b) The case when $m$ is even and $s$ is odd is analogous to case (Ia), i.e. the sum of the first, second and forth summand is zero:

$$
\begin{gathered}
L(k, m, n)=\Gamma(s-1)(b+c-a+(w-u)(w-v)) \\
+(v-w) \Gamma(s-1)(w-u)+c \Gamma(s-3) b(2 c-a)-b \Gamma(s-2)(b+c-a)=\Gamma(s-2) b(2 c-a) .
\end{gathered}
$$

c) Now assume that $m$ is odd and $s$ is even. Then

$$
\begin{gathered}
L(k, m, n)=\Gamma(s)(w-u)+(v-w) \Gamma(s-1)(b+c-a) \\
+c \Gamma(s-1)(w-u)-b \Gamma(s-1)(v-u)=\Gamma(s-1)(a(w-v)+c(v-u)) .
\end{gathered}
$$

d) Finally, if $m, s$ are odd then

$$
\begin{gathered}
L(k, m, n)=\Gamma(s)(w-u)+(v-w) \Gamma(s-2) b(2 c-a) \\
+c \Gamma(s-1)(w-u)-c \Gamma(s-2)(a(w-v)+c(v-u))=\Gamma(s)(v-u),
\end{gathered}
$$

which completes the whole proof.

COROLlaRY. The sequence $\left\{P_{n}\right\}_{n=0}^{\infty}$ admits nonnegative product linearization if and only if:

$$
\begin{aligned}
& a \leq b+c, \quad a \leq 2 c, \quad a \leq b+c+(w-u)(w-v) \\
& u \leq v, \quad u \leq w, \quad \text { and } \quad 0 \leq a(w-v)+c(v-u) .
\end{aligned}
$$

For example, if either $w=v$ or $w=u$ then $\left\{P_{n}\right\}_{n=0}^{\infty}$ admits nonnegative product linearization if and only if $a \leq b+c, a \leq 2 c$ and $u \leq v$.

Finally let us specify our results to orthogonal polynomials related to limit measures with respect to conditionally free indendence introduced by Bożejko, Leinert and Speicher [BLS].

1. The central limit theorem (Theorem 4.3 in [BLS]): $a=\alpha^{2}, b=c=\beta^{2}, u=v=$ $w=0$. Here $\left\{P_{m}\right\}_{m=0}^{\infty}$ admit nonnegative product linearization if and only if $\alpha^{2} \leq 2 \beta^{2}$ and this holds if and only if the corresponding measure has no atom.

2. The Poisson limit theorem (Theorem 4.4 in [BLS]): $a=\alpha^{2}, b=c=\beta^{2}, u=\alpha^{2}$, $v=w=\beta^{2}+1$ (these recurrence coefficients are not calculated explicitly in [BLS] but they can be derived from the final formula in $[\mathrm{M}])$. Here the necessary and sufficient condition for nonnegative product linearization for these polynomials $\left\{R_{n}\right\}_{n=0}^{\infty}$ is $\alpha^{2} \leq \beta^{2}+1$ and $\alpha^{2} \leq 2 \beta^{2}$.

3. Let us now consider the symmetrization of the Poisson measure. It means that we are dealing with polynomials $\left\{Q_{n}\right\}_{n=0}^{\infty}$ which are related to $\left\{R_{n}\right\}_{n=0}^{\infty}$ by $R_{n}\left(x^{2}\right)=Q_{2 n}(x)$, so we get $a=\alpha^{2}, b=1, c=\beta^{2}$ and $u=v=w=0$. Here the corresponding condition is the same as in the previous case: $\alpha^{2} \leq \beta^{2}+1$ and $\alpha^{2} \leq 2 \beta^{2}$. 


\section{References}

[As] R. Askey, Linearization of the product of orthogonal polynomials, in: Problems in Analysis, R. Gunning (ed.), Princeton University Press, Princeton, New Jersey, 1970, $223-228$.

[Ch] T. Chihara, An Introduction to Orthogonal Polynomials, Mathematics and Its Applications 13, Gordon and Breach, New York, 1978.

[BH] W. R. Bloom and H. Heyer, Harmonic Analysis of Probability Measures on Hypergroups, de Gruyter Studies in Mathematics 20, Berlin, 1995.

[BLS] M. Bożejko, M. Leinert and R. Speicher, Convolution and limit theorems for conditionally free random variables, Pacific J. Math. 175 (1996), 357-388.

[Ko] T. H. Koornwinder, Discrete hypergroups associated with compact quantum Gelfand pairs, in: Applications of Hypergroups and Related Measure Algebras, W. Connett et al. (eds.), Contemp. Math. 183, Amer. Math. Soc., 1995, 213-237.

[M] W. Młotkowski, Free probability on algebras with infinitely many states, Probability Theory and Related Fields 115 (1999), 579-596.

[MS] W. Młotkowski and R. Szwarc, Nonnegative linearization for polynomials orthogonal with respect to discrete measures, Constructive Approximation 17 (2001), 413-429.

[SY] N. Saitoh and H. Yoshida, The infinite divisibility and orthogonal polynomials with a constant recursion formula in free probability theory, Probability and Mathematical Statistics 21 (2001), 159-170.

[Sz1] R. Szwarc, Orthogonal polynomials and a discrete boundary value problem, I, SIAM J. Math. Anal. 23 (1992), 959-964.

[Sz2] R. Szwarc, Orthogonal polynomials and a discrete boundary value problem, II, SIAM J. Math. Anal. 23 (1992), 965-969. 